\title{
Acuerdos oligopolísticos entre las dos principales empresas de un sector. El caso de MZA y NORTE
}

\author{
- Miguel Ángel Villacorta Hernández \\ Universidad Complutense de Madrid
}

\section{Introducción}

El 31 de julio de 2013 se liberalizó el tráfico ferroviario privado de personas en España, de forma que desde ese momento desapareció el monopolio estatal del sector y comienzan a competir empresas públicas y privadas. La Red Nacional de los Ferrocarriles Españoles (RENFE) fue una empresa estatal española que se encargó del transporte de pasajeros y mercancías por la red nacional ferroviaria de ancho ibérico desde su creación, el 24 de enero de 1941, a su disolución, el 31 de diciembre de 2013. ${ }^{1}$ Únicamente en esos sesenta y dos años existió en España una empresa pública que mantuvo el monopolio de toda la red ferroviaria de ancho ibérico, ${ }^{2}$ por el contrario, un período de tiempo más extenso, desde el primer ferrocarril en 1847 hasta 1941, y a partir de 2013, las compañías ferroviarias han sido y son privadas. Por todo ello, el estudio de las compañías ferroviarias privadas es una forma de estudiar el pasado y el futuro.

La investigación se centra en la Compañía de los Caminos de Hierro del Norte de España (NORTE) y la Compañía de Madrid a Zaragoza y Alicante (MZA), porque son las dos empresas españolas con mayor dimensión de la época, ${ }^{3}$ tanto por el peso de sus activos, como por la mano de obra emplea-

1. A partir del 31 de diciembre de 2013, RENFE se dividió en dos entidades: ADIF, ente gestor de las infraestructuras ferroviarias españolas, y Renfe Operadora, compañía que se quedó con la explotación de los ferrocarriles de titularidad pública.

2. Nos referimos a red de ancho convencional en toda la red salvo la de vía estrecha. La primera se nacionalizó en RENFE, la segunda se agrupó posteriormente en FEVE.

3. Las dos compañías se constituyeron como sociedades anónimas en un intervalo de dos años de diferencia. MZA se constituyó el 31 de diciembre de 1856 (R. D. de 26 de enero

Fecha de recepción: noviembre 2017

Versión definitiva: diciembre 2018

Revista de Historia Industrial

N. ${ }^{\circ} 75$. Año XXVIII. 2019 
da, e incluso fueron las mayores privadas españolas hasta que llegaron los procesos de privatización y de fusiones continuas de finales del siglo XX.

NORTE fue la empresa privada más importante de España, tanto por el importe de sus activos, como por el número de kilómetros en explotación, o por el número de trabajadores, siendo la segunda MZA en todos estos indicadores. Dentro de las empresas europeas, NORTE pasó de ser la decimoctava empresa de Europa por volumen de activos en 1890, a ser la décima del continente a partir de 1917 (Carreras y Tafunell, 1993, pp.127-174; 1996, p. 77; 1997, pp. 277-304) (Carreras, 1999, p. 39). Las mismas citas muestran como MZA pasó de ser la decimonovena a ser la undécima de Europa en los mismos años, siempre siguiendo los pasos por detrás de NORTE, pero muy cerca.

Ambas suponían el $63,41 \%$ de las líneas explotadas, ${ }^{4}$ por lo que se puede inferir que, estudiando las dos conjuntamente, se analiza gran parte del sector. Además, su indiscutible relevancia en el panorama de los ferrocarriles europeos permite observar la situación de las grandes empresas europeas en esa época.

Las dos compañías alcanzaron interesantes acuerdos empresariales: tres oligopolísticos de gestión para luchar contra el mercado y el Estado, uno en el que se alcanzó una dirección unificada desde 1906 e, incluso, estuvieron muy cerca de fusionarse en tres ocasiones.

Esta investigación analiza la perspectiva comparada de NORTE con MZA, y esto anteriormente había sido realizado por Tedde (1978, cap. 1), Cordero y Menéndez (1978, pp. 163-338), Comin et al. (1998), Ortúñez (1999a) (1999b), Vidal y Ortúñez (2002) y de los Cobos y Martínez Vara (2009). En concreto, este artículo analiza los acuerdos de colaboración comercial entre ambas compañías. Con anterioridad solo había sido analizado alguno de ellos individualmente al estudiar otros temas, sin que se hubieran descrito todos los pactos de forma conjunta.

El artículo comienza analizando la evaluación de la competencia entre ambas compañías, para continuar describiendo cada uno de los pactos toma-

\footnotetext{
de 1857 autorizando a la constitución definitiva de la Compañía de Ferrocarriles de Madrid a Zaragoza y Alicante, Ministerio de Fomento. Vid. Colección Legislativa de la Deuda española / Colección de Decretos). NORTE quedó inscrita el 29 de diciembre de 1858 y se obtuvo la constitución definitiva y aprobación de los estatutos el 14 de enero de 1859 (R. D. de 14 de enero de 1859 autorizando a la constitución y aprobación de los estatutos de la Compañía de los Caminos de Hierro del Norte de España, Ministerio de Fomento (Colección Legislativa de la Deuda española/Colección de Decretos).

4. En 1923, MZA explotaba 3.663 kilómetros-2.944 de la Antigua Red y 719 de la Red Catalana (De la Torre, 1923, p. 101; MZA, 1924, p. 4). En 1923, NORTE explotaba 3.692 kilómetros (De la Torre, 1923, p. 65; NORTE, 1924, p. 5). En ese mismo ejercicio económico, en España existían 11.599 kilómetros de vía normal - 11.559 a vapor y 40 eléctricos- (De la Torre, 1923, p. 62). El porcentaje de $63,41 \%$ ha sido calculado por el cociente $7.355 / 11.599$. Tedde $(1978$, p. 11) va más lejos cuando afirma que ambas compañías constituían el $90 \%$ del tendido ferroviario con tráfico de cierta intensidad.
} 
dos entre ambas. La conclusión esperada es indagar sobre la relevancia de los acuerdos y alianzas.

En la investigación se ha realizado un análisis, tanto de fuentes primarias, compuestas por la legislación, documentación y correspondencia emitida por la compañía NORTE y MZA, como por las fuentes secundarias escritas por autores y organizaciones. La fuente principal son los diversos documentos referidos al tema que se conservan en el Archivo Histórico Ferroviario (AHF) de la Fundación de Ferrocarriles Españoles con sede en Madrid. Respecto a las fuentes secundarias, en el trabajo se analizan fuentes de la época de las concesionarias privadas del ferrocarril, pero, además, también se observan los resultados de las investigaciones posteriores sobre el ferrocarril.

La investigación requiere la comparación temporal de datos contables, para la que se han realizado cuatro figuras, en las cuales ha sido necesario corregir la inflación. Para corregir el efecto de las variaciones en los precios, todos los valores monetarios han sido referidos al año base 1913 utilizando los índices de coste de la vida que aparecen en las tablas del trabajo de Balleste$\operatorname{ros}\left(1997\right.$, pp. 363-395). ${ }^{5}$

\section{Relación competencial entre MZA y NORTE}

Los problemas económicos de las ferroviarias tuvieron su origen en las deficiencias estructurales, pues su negocio requería de unos altos costes iniciales de puesta en marcha, con altas inversiones en vías y material, y altas necesidades de recursos humanos. Para compensar tantos esfuerzos económicos, el negocio requería un número elevado de viajes, ya fuera de personas o de materiales. Sin embargo, la situación económica del país era mala; las empresas no encontraban la demanda suficiente para transportar sus productos y la mayoría de las familias no tenían cultura de turismo ni el poder adquisitivo necesario para viajar frecuentemente.

La estrategia óptima de las compañías hubiera sido la de especializarse en nichos de mercado rentable: cereales de Castilla al Mediterráneo, de la industria barcelonesa o vasca al resto de España o llevar a todo el país los productos de la huerta mediterránea. Con todo, el miedo a perder posición competitiva hizo que todas las empresas, en mayor o menor medida, tomaran el camino equivocado de intentar absorber a otras más pequeñas. De esta forma, las empresas concesionarias del ferrocarril en España, especialmente MZA y NORTE, llegaron a tener una estructura empresarial muy desajustada: presentaban varias líneas poco rentables, con una herencia de altos costes

5. Martínez Vara (2004, p. 112) hizo un comentario sobre los índices de precios en relación con su aplicación a las ferroviarias. 
de construcción, con muchos empleados y, además, con mala planificación, porque la proporción de empleados contratados directamente por ellas era baja en comparación con los obtenidos por la absorción de otras compañías.

Ante la mala situación económica general del país, de las empresas y de las familias, la única manera de mejorar la cuenta de resultados era aumentar las tarifas o recibir más ayudas por la compra de material y el mantenimiento del personal. El Estado no tenía los recursos suficientes para aumentar las tarifas y, por si fuera poco, existía una corriente de pensamiento social que proponía que también se eliminaran los anticipos de personal y material.

La única forma que encontraron las ferroviarias para sobrevivir era que se incrementasen las ayudas gubernamentales, aunque fuera a costa de aumentar la intervención estatal en ellas. Este parecía el único camino para el Estado y para ellas, y es el que se tomó.

No obstante, había otro camino. En este escenario las ferroviarias españolas buscaron apoyos entre ellas para aunar esfuerzos, normalmente contra el Estado. ${ }^{6}$ Así lo hicieron las tres empresas ferroviarias más importantes en algunas ocasiones, ${ }^{7}$ y con mucha mayor frecuencia y relevancia las dos grandes.

Las prácticas estratégicas de actuación conjunta entre NORTE y MZA se iniciaron en sus procesos de absorción del período 1874-1896 (Vidal Olivares, 1999 , p. 643). Los principales acuerdos fueron tomados sobre las tarifas nacionales; sin embargo, también las compañías comenzaron a plantear acuerdos en sus relaciones internacionales, a medida que aumentaron los vínculos con los mercados exteriores, especialmente los de Europa occidental y los de Portugal (Vidal Olivares, 1995a).

NORTE y MZA tuvieron un acercamiento y colaboración profunda. Una de las posibilidades de las dos grandes empresas de capital francés para mejorar su situación económica era fortalecer su posición frente al gobierno y frente al mercado. Con ella se pretendía lograr, entre otros objetivos, la reducción de los costes de explotación. Hubo cuatro momentos de especiales dificultades económicas de la explotación, en los que se tomaron caminos de acercamiento entre ambas empresas: los acuerdos comerciales para actuar como oligopolio entre MZA y NORTE de 1870, 1875, 1896 y, el más impor-

6. Muchos son los ejemplos de estas alianzas: NORTE y MZA $(1934 ; 1933 ; 1932 ; 1916$; $1915 ; 1902)$, NORTE, MZA, Andaluces y MCP $(1922 ; 1919)$ y NORTE, MZA, Andaluces, MCP y Oeste (1907, pp. 1-31).

7. La primera posición conjunta relevante de las tres grandes - NORTE, MZA y Andaluces - fue la reclamación de la Federación Nacional de los Ferroviarios Españoles derivadas del Primer Congreso Ferroviario de junio de 1912. Las características de esta alianza pueden observarse en: Carta de Federación Nacional de los Ferroviarios Españoles al Director de la Compañía de Ferrocarriles Andaluces: 20 julio de 1912. Carta del presidente del Consejo de Administración de la Compañía de Ferrocarriles Andaluces al Consejo de Administración de NORTE: 26 de julio de 1912. Acta n. ${ }^{\circ} 803$ del Libro de actas del Consejo de Administración de NORTE: 28 de septiembre de 1912, Apartado III.9. 
CUADRO 1 - Tipo de vinculación entre MZA y NORTE

\begin{tabular}{lll}
\hline Fecha & \multicolumn{1}{c}{ Tipo de acuerdo } & Empresas participantes \\
\hline 1870 & Acuerdo de oligopolio. Convenio de tarifas & MZA y NORTE \\
\hline 1875 & Acuerdo de oligopolio. Convenio de distancia más corta & MZA y NORTE \\
\hline 1896 & Acuerdo de oligopolio. Convenio de reparto de tráfico & TBF, MZA y NORTE \\
\hline 1906 & Acuerdo de oligopolio. Sindicatura & MZA y NORTE \\
\hline
\end{tabular}

Fuente: Elaboración propia.

tante (Sindicatura), de 1906. El cuadro 1 recoge el tipo de vinculación entre NORTE y MZA.

Los acuerdos de oligopolio de 1870, 1875, 1896 y 1906 fueron propuestos por NORTE y se llevaron a cabo porque MZA sí aceptó. Por su parte, el oligopolio de tres empresas entre MZA, NORTE y Andaluces y la posterior fusión de $1923^{8}$ no partió de ninguna ferroviaria, sino que fue primeramente propuesta por el Estado, y posteriormente matizada por el propio Estado; en este caso, la negativa a la propuesta de oligopolio partió de NORTE, y la negativa a la fusión vino de las tres empresas, sobre todo porque nunca llegaron las prometidas ventajas económicas y fiscales para fomentar la fusión.

\section{Enfrentamiento inicial de MZA-NORTE}

Las luchas comerciales, productivas y estratégicas de NORTE y MZA produjeron un inicial escenario de lucha competitiva.

La historia de la competencia empresarial entre ambas puede dividirse en tres etapas. Entre 1855-1873 eran dos de las compañías de mayor envergadura financiera y distributiva, pero con parecido comportamiento en el mercado que otras sociedades ferroviarias españolas. Entre 1874 y 1905, la estrategia expansionista de las dos sociedades llegó a plantear abiertamente un conflicto comercial entre ambas para controlar el tráfico. A partir de 1906, NORTE y MZA consagran un régimen de duopolio con reparto de beneficios y comienza un proceso de integración entre ambas compañías.

En la primera etapa se produjeron movimientos competenciales y litigios por medio de empresas satélites, controladas por ellas y que, posteriormente, se integrarían en los dos gigantes ferroviarios españoles. Las más importantes

8. Desde los primeros intentos de ordenación ferroviaria, los gobiernos españoles creían que la mejor alternativa para los intereses nacionales era que las líneas ferroviarias se reuniesen en pocas compañías. Así incluso lo expresaron el presidente del Consejo de Ministros de España, Ramón María Narváez, y su ministro de Fomento, Manuel de Orovio (Wais, 1948, p. 115). 
tuvieron lugar en la provincia de Barcelona. En 1885, existió una reivindicación de la Compañía del Norte de Barcelona a Granollers (posteriormente de MZA) en contra de la Compañía del Ferrocarril de Barcelona a Zaragoza (posteriormente de NORTE) debido a la rivalidad surgida entre ambas. ${ }^{9}$

El período en el que MZA y NORTE compitieron más duramente fue 1874-1896. NORTE llevó a cabo una estrategia de absorciones y fusiones con la intención de realizar una ofensiva para mejorar su posición frente a MZA ${ }^{10}$ en el mercado ferroviario español. Tanto la integración del ferrocarril de Alar a Santander en 1874, como la incorporación a la explotación de la Compañía de Zaragoza a Pamplona y Barcelona en 1878, y la del ferrocarril de Tudela-Bilbao en 1879, tenían como finalidad cerrar espacios en el País Vasco para intentar dominar el tráfico en la zona.

El siguiente movimiento estratégico de NORTE fue la apertura de influencia hacia la fachada mediterránea, donde dominaba MZA. Ambos colosos disputaban por integrarse a la rentable Compañía de Almansa a Valencia y Tarragona, propiedad del grupo del financiero y banquero valenciano José Campo. Aunque las dos compañías estaban interesadas en hacerse con su control, hasta el fallecimiento de Campo en 1890 no comenzaron las negociaciones. Finalmente, NORTE la adquirió en 1891. Con la adquisición de la Compañía de Almansa a Valencia y Tarragona, NORTE asestó un duro golpe a MZA, pues se introdujo de lleno en un mercado mediterráneo en el que hasta entonces MZA había actuado como monopolista. La estrategia agresiva de NORTE en la región valenciana continuó con la adquisición en 1892 de la Compañía del Este de España y del ferrocarril Valencia-Utiel.

Como respuesta a estos movimientos de NORTE, MZA construyó en 1895 la línea de Ariza, que permitía a sus trenes penetrar en el corazón del imperio castellano de NORTE. Esta rivalidad supuso una guerra de tarifas entre ambas compañías. ${ }^{11}$

9. La Compañía del Ferrocarril de Barcelona a Zaragoza sostenía que tenía el derecho a prolongar la línea que, arrancando en Sabadell terminara en Moncada y desde este punto prolongar hasta Barcelona. Por el contrario, la Compañía del Ferrocarril de Barcelona a Granollers defendía el derecho a impedir esta prolongación y a obligar a dicha línea a que siguiera empalmando en Moncada con su ferrocarril. Esta compañía alegaba que, además, había sido construida sin subvenciones, por lo cual no era lícito interceptarle lucros que le corresponderían por afluencias naturales de la línea y que se favorezca en contra una empresa subvencionada (Camino de Hierro del Norte (1855, pp. 1-23).

10. Esta finalidad fue observada por Vidal Olivares (1997, p. 52).

11. La guerra de tarifas entre MZA y NORTE fue estudiada por Gómez Mendoza (1982, p. $5 ; 1989$, p. 62$)$. 


\section{Convenio de tarifas MZA-NORTE en 1870}

En algunas ocasiones, NORTE y MZA aparcaron su antigua y dura competencia, concluyendo diversos pactos y acuerdos para obtener mejores rendimientos de las explotaciones. ${ }^{12}$ Los pactos interempresariales estuvieron favorecidos por las características de la industria de las concesionarias del ferrocarril, propia de una estructura oligopolística: homogeneidad del producto, pocos productores, existencia de barreras de entrada y control de mercado. Los miembros de un oligopolio son susceptibles de establecer acuerdos entre ellos para proceder al reparto del mercado y para mantener un sistema común de tarifas. La finalidad de los pactos de los oligopolios era intentar incrementar los beneficios conjuntos de los socios porque, al menos en teoría, estos beneficios serán siempre superiores a la suma de los beneficios individuales de cada empresa si estas deciden operar en condiciones libres.

El primer acuerdo entre ambas compañías fue el de convenios tarifarios de 1870, que se produjo por dos razones básicas. La primera es que en este momento se produce el término de la primera fase de la construcción de las líneas de ambas compañías y en consecuencia se produce la conexión de la red básica peninsular; en esta situación fue necesario acordar tarifas de intercambio y de transbordo entre ellas. La segunda, que subyace a la anterior, es la necesidad de desarrollar los tráficos de modo más armónico para aumentar los rendimientos de la red de ambas compañías y hacer frente a los problemas de rentabilidad ocasionados por los problemas financieros de 1865-1869.

Las dificultades financieras de MZA y NORTE comenzaron en 1865, pero fueron agravadas por la lucha salvaje llevada entre ellas. Entre 1866 y 1871, el aumento de cargas financieras (Figura 1) y el estancamiento de los ingresos de explotación (Figura 2) provocaron resultados contables negativos (Figura 3). Estas dificultades se agudizaron con la Revolución de 1868, que provocó el exilio de la reina Isabel II y significó el comienzo del Sexenio revolucionario. Durante este período, la crisis ferroviaria se agudizó, provocando la quiebra y desaparición de numerosas compañías. MZA y NORTE lograron resistir las adversidades de esta época, aprovechando la quiebra de las pequeñas empresas para ir expandiendo sus negocios y crecer en tamaño (Wais, 1974, p. 192) hasta convertirse en las dos mayores empresas ferroviarias del país. En gran parte, NORTE y MZA salvaron la situación por haber firmado el acuerdo sobre la distribución del tráfico de 1870. Este convenio se consumó porque MZA quiso, pues en NORTE siempre desearon alcanzar este tipo de acuerdos. Y ahora podemos afirmar que MZA tomó la decisión correcta porque el acuerdo le salvó de esa gran crisis, tanto que le permitió dar incluso dividendos en el año 1873.

12. Más adelante los acuerdos se tomaron también para competir contra las líneas de transportes por carretera (García Venero, 1948, p. 353). 
Las posturas entre MZA y NORTE se fueron acercando paulatinamente, aunque se puede observar un salto en su evolución con la actuación de Barat en NORTE.

Jean Barat ${ }^{13}$ fue director general entre el 20 de febrero de 1881 y el 30 de abril de 1901. Barat dirigió y coordinó los procesos de fusión y adquisición con las diversas empresas. En los años anteriores a 1870, organizó la estructura financiera de NORTE tras los descalabros experimentados en los años de la crisis de 1868, llevando a cabo una restructuración de las obligaciones en circulación. Además, durante su gestión en NORTE, fue el artífice de las relaciones comerciales con los competidores, especialmente en lo que se refiere a convenios de tarifas con MZA en 1870 y en 1896, y el de la distancia más corta de 1875.

Como jefe comercial, en 1870, Barat planteó el convenio de tarifas con MZA basado en el abono de una cantidad alzada, pagadera por años, en lugar del proyecto de sindicatura de productos o de porcentaje por tonelada propuesto por el entonces director Noblemaire. Este convenio puso fin a la lucha empresarial que NORTE y MZA venían soportando, por el tráfico desde Bilbao y Alsasua a Madrid y hacia otros destinos meridionales a través del empalme de Casetas, en Zaragoza — punto de entronque con las líneas de MZA-(Vidal Olivares, 1999, p. 643). Este acuerdo fue planteado por Barat, pero el que estaba en peor situación era MZA, que por eso aceptó.

\section{GRÁFICO 1 - Cargas financieras de NORTE y MZA en millones de pesetas}

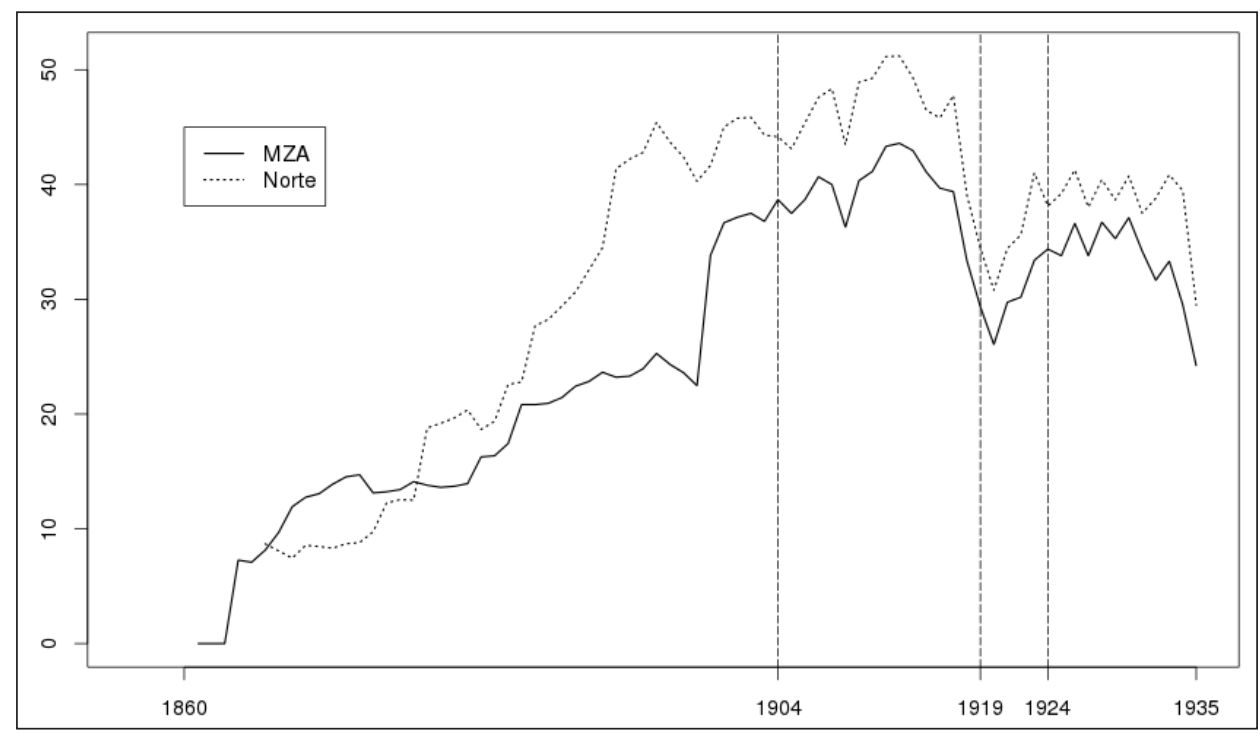

Fuente: Elaboración propia.

13. Su biografía fue estudiada por Wais (1987, p. 196). 
GRÁFICO 2 - Ingresos de explotación de NORTE y MZA en millones de pesetas

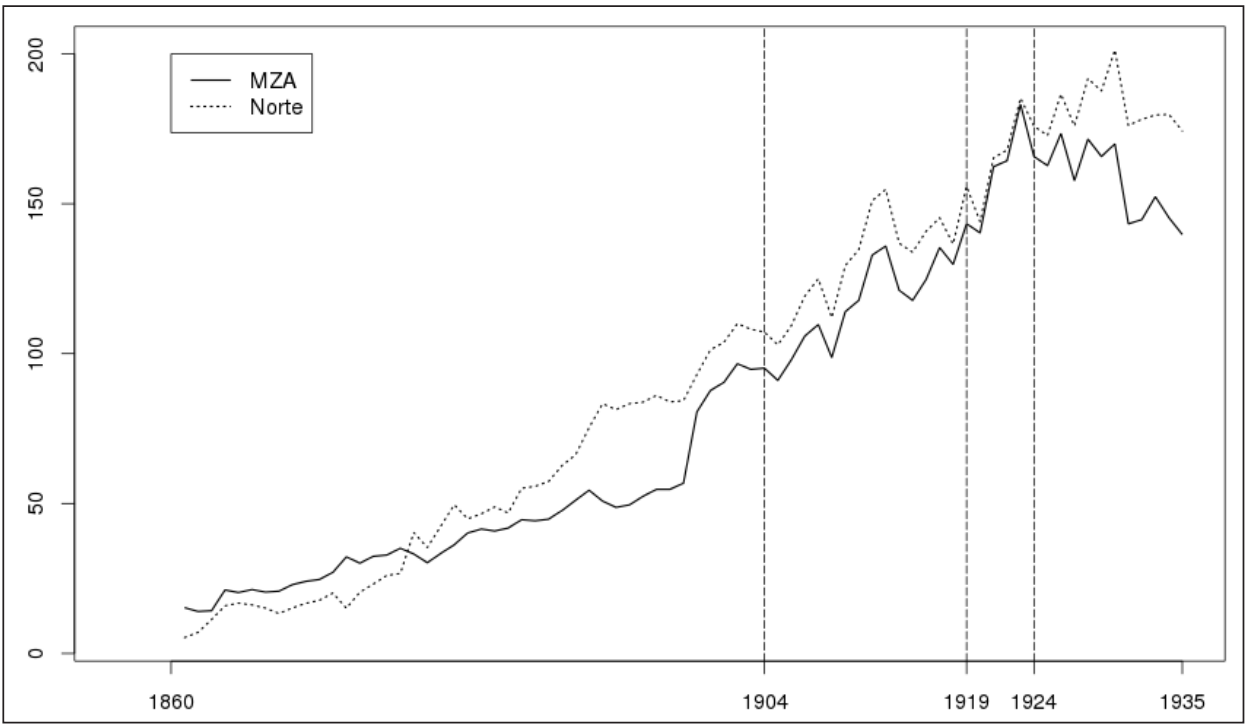

Fuente: Elaboración propia.

GRÁFICO 3 - Resultado contable final de NORTE y MZA en millones de pesetas

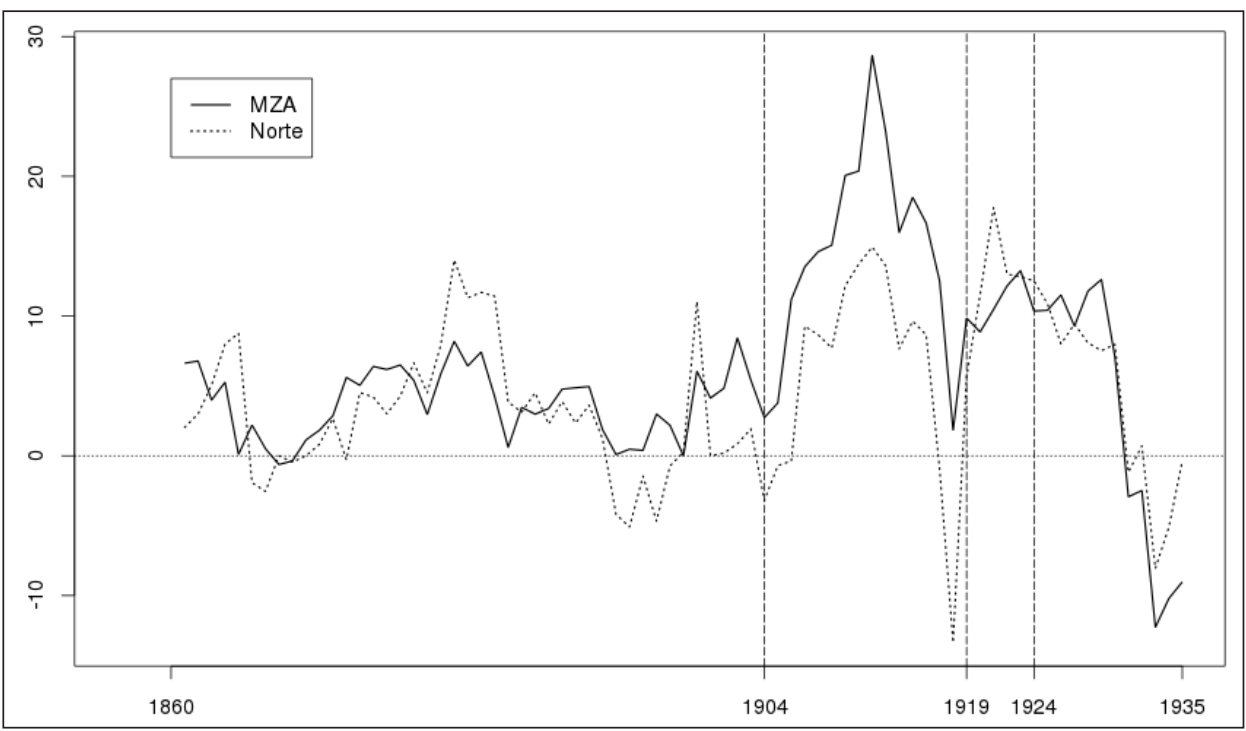

Fuente: Elaboración propia. 


\section{Acuerdo de la distancia más corta MZA-NORTE de 1875}

La habilidad desarrollada por Barat en las negociaciones para establecer el primer convenio de tarifas con MZA, le llevó a ser nombrado subdirector de NORTE. Desde este cargo continuó llevando el peso de la estrategia comercial de la compañía, que estaba dando buenos resultados. Barat, como subdirector, planteó un acuerdo decisivo con MZA: el convenio para la división del tráfico entre las dos redes bajo la base y el principio de la distancia más corta.

El principal desencadenante de este nuevo acuerdo entre ambas fue la maniobra de MZA, cambiando su política estratégica hacia una actitud más colaboradora. El segundo desencadenante fue el fallecimiento de Émile Pérei$\mathrm{re}^{14}$ en enero de 1875 , que facilitó el acuerdo tan solo mes y medio después.

Tras la crisis financiera de 1865-1869 MZA cambió su estrategia. A partir de ese momento no pidió nuevas concesiones, sino que su expansión se realizó por medio del rescate y fusión con compañías prexistentes. Además, buscó alianzas y acuerdos con NORTE lo que supuso una forma de reconciliarse con ella. El 5 de octubre de 1875, MZA adquirió la Compañía del Ferrocarril de Córdoba a Sevilla y sus 132 kilómetros de líneas ya en explotación así como la línea de las minas de la Reunión a Villanueva del Río. A cambio, MZA permitió la anexión de la Compañía de los Ferrocarriles de Zaragoza a Pamplona y Barcelona (ZPB) por NORTE (Cameron, 1961, p. 253). La fusión entre NORTE y ZPB pudo realizarse en 1874, pero el acuerdo no se alcanzó porque eran contrarios los socios catalanes, predominantes en el Consejo de ZPB, y porque había dos administradores - Ros Olano y Llorente- que lo eran simultáneamente de ZPB y MZA. ${ }^{15}$ Pero todo esto cambió tras el acuerdo de $1875 .{ }^{16}$

Las empresas habían mantenido sus alianzas, pero tuvieron un encontronazo por fuertes litigios por las cargas entre el puerto de Bilbao y Madrid. Para solventarlo, el 8 de marzo de 1875, NORTE y MZA acordaron repartirse los tráficos en esas líneas por medio del principio de que circularían por la «más corta distancia». Por el acuerdo se estableció que las mercancías debían circular como norma por la línea que demostrase menor kilometraje entre origen y destino, ${ }^{17}$ de esta forma, siempre que un tráfico debiese circular por uno o por los dos ferrocarriles, lo haría por el camino más corto en kilómetros. ${ }^{18}$

14. Rondo E. Cameron (1961) comparte la opinión de que la muerte de Émile Péreire facilitó el acercamiento entre las dos grandes ferroviarias españolas.

15. Cartas de Bauer 6-8-1874, 9-8-1874 y 21-8-1874. Archivos Rothschild London. Estas cartas fueron estudiadas por López Morel (2005, p. 292).

16. Más adelante, NORTE llegó al acuerdo con MZA, lo que permitió la fusión de NORTE con ZPB, que se hizo efectiva el 13 de febrero de 1878 .

17. Una estrategia similar había sido tomada en 1863 entre «Midi, Paris a Lyon et a la Méditerranée y Po» (Caron, 1997).

18. Este acuerdo fue analizado por Gómez Mendoza (1989, p. 59), Comín et al. (1998, vol. I, p. 216) y López-Morel (2005, p. 478). 
Gracias al acuerdo, MZA y NORTE establecieron una zona de influencia para cada una de ellas, se aseguraron el tráfico de su área y, al no establecerse competencia entre ellas, pudieron, además, aplicar tarifas elevadas.

\section{Convenio de reparto de tráfico NORTE-MZA-TBF en 1896}

El acuerdo para circular por la vía más corta de 1896 fue derivado del acuerdo de 1875. El objeto inicial del acuerdo por el principio de la distancia más corta de 1875 tenía por objeto el trayecto hacia el puerto de Bilbao, y se fue al traste por un enfrentamiento entre MZA y NORTE por el tráfico entre Castilla y Cataluña a propósito de la apertura de la línea de Valladolid a Ariza y la variante de Caspe. Esta línea ponía a MZA en contacto directo con Barcelona y Zaragoza: «... la compañía de Alicante, unida así en Zaragoza con la de Tarragona a Barcelona y Francia, estableció un sinnúmero de tarifas, vía Caspe, con precios más reducidos que los que regían anteriormente en combinación con la línea de NORTE, desviando de esta última entre Zaragoza y Barcelona, vía Manresa, el tráfico de viajeros y mercancías que la utilizaban». ${ }^{19}$ La pugna de tarifas fue dura,${ }^{20}$ por lo que Barat planteó a MZA un entendimiento en diciembre de 1894 que no fue aprobado, lo cual ahondó en la «guerra de tarifas» hasta que, finalmente, el 4 de enero de 1896, se firmó un acuerdo parecido al que había presentado Barat. ${ }^{21}$

El convenio sobre reparto de tráficos, firmado el 4 de enero de 1896, por MZA, NORTE y TBF (Tarragona, Barcelona y Francia), ${ }^{22}$ tuvo una duración de diez años (MZA, 1896a, p. 4). Este acuerdo es «un convenio que pone término a las rivalidades y que concilia los intereses de las tres compañías» (MZA, 1896b, p. 4).

Este convenio no supone un acuerdo global entre ambas compañías. Los pactos son únicamente sobre corrientes de tráficos entre Barcelona, Lérida, Zaragoza y Valladolid, no en otros tramos.

Según este acuerdo se aplicaron precios totales iguales al transporte en tres tramos: entre Barcelona y Zaragoza, entre Zaragoza y Valladolid y entre

19. Carta enviada por J. Barat el 4 de abril de 1908 al presidente del Consejo de Administración de NORTE (p. 14), realizando una exposición de las indemnizaciones que la compañía le debía.

20. En concreto la pugna de tarifas fue muy dura en lo que se refiere al trigo, las harinas y los vinos (Sanz, 1985) (Gómez Mendoza, 1984).

21. La firma del acuerdo de 1896 fue propiciado por Barat, y firmado por Sagasta y Elduayen por NORTE, el Marqués de Urquijo y Baüer por MZA y Planas por TBF (Tarragona, Barcelona y Francia).

22. El 1 de enero de 1898 MZA absorbió la Compañía de Tarragona a Barcelona y Francia (TBF), denominada desde entonces «Red Catalana de MZA», que había suspendido pagos y explotaba 723 kilómetros. 
Lérida y Barcelona (por Manresa y Picamoixons). Hasta ese momento existía la posibilidad de recorrer esos tramos por las líneas de las dos compañías, dejando a los clientes la capacidad de elegir la vía. Las recaudaciones del tráfico de dichas líneas formarían un fondo común entre MZA, NORTE y TBF que se repartiría entre ellas tras haber deducido los gastos y aplicando coeficientes de reparto.

En la vigencia de este acuerdo de 1896, existieron más ejemplos en la colaboración NORTE y MZA. Los dos más importantes se produjeron en 1902. Una carta oficial del Comité de Barcelona de NORTE, firmada por el señor Girona, propuso la construcción de una estación común entre NORTE y MZA $;{ }^{23}$ el presidente de NORTE decidió incluirla en el acta del Consejo. ${ }^{24}$ De la misma forma, NORTE y MZA (1902, p. 1-16) publicaron el documento «Contrato y reglamento celebrados entre la Compañía de los Caminos de Hierro del Norte y la de los Ferrocarriles de Madrid a Zaragoza y a Alicante para el cambio de material». En él se recogían las condiciones establecidas entre ambas compañías para regular la circulación de material de una compañía en las líneas de la otra. En el contrato se fijaban los transbordos que debía hacer cada coche o furgón, recargos por coches de lujo y alquiler de material con precios especiales. Los vagones de mercancías no estaban sujetos a transbordo y circularían libremente si estaban en buenas condiciones, siendo utilizados a su regreso por la compañía por cuya línea circulan. Además, el contrato especificaba el establecimiento de saldos, compensaciones, devoluciones y registros.

\section{Convenio «Sindicatura general de productos» NORTE-MZA: Comisión Mixta de 1906}

Con el acuerdo de 1870 se inició una fase de entendimiento entre NORTE y MZA; el acuerdo de 1870 fue renovado en 1896, y posteriormente sustituido por otro, el más importante, en 1906.

En el Informe Anual de 1905, NORTE (1906, p. 34) explica que «durante el ejercicio de 1905 nuestras relaciones con las demás compañías de ferrocarriles se han mantenido en los mismos términos de cordialidad que en los anteriores, proponiéndose estrechar aún más la comunidad de intereses que nos liga con la de Madrid a Zaragoza y a Alicante; propósito que, al realizarse, permitirá mejorar y perfeccionar los servicios en beneficio del público, reportando al mismo tiempo positivas ventajas a las dos compañías». En el Infor-

23. Gaceta de los Caminos de Hierro, 16 de febrero de 1908.

24. Acta n. ${ }^{\circ} 674$ del Libro de actas del Consejo de Administración de NORTE: 21 de marzo de 1902. 
me Anual de 1906 de NORTE (1907, p. 33) aparece que «desde el ejercicio de 1905 existen unas excelentes relaciones entre MZA y NORTE; relaciones que han dado como resultado un completo acuerdo entre ambas compañías». En el mismo documento, la empresa afirmaba que este acuerdo daría como resultado una mayor rapidez en los transportes, una simplificación de las tarifas y, sobre todo, las compañías obtendrían la apreciable ventaja de no tener que preocuparse de las diferentes direcciones que pudiera tomar el tráfico.

El acuerdo entre NORTE y MZA, denominado de «Sindicatura general de productos», se tomó el 27 de junio de $1906,{ }^{25}$ cuando MZA ya había absorbido totalmente a TBF. ${ }^{26}$ En ese momento se estableció una duración de veinticinco años, desde el 1 de enero de 1907 hasta el 31 de diciembre de 1931. El convenio sería denunciable por cualquiera de las dos compañías con una antelación de dos años. El contrato se mantuvo hasta 1925, cuando dejó de estar vigente.

El Consejo de NORTE designó a los administradores Borregón y Estibans para que firmasen el Convenio en representación de la compañía, así como para formar parte de la Comisión establecida en el artículo 14 del Convenio, «encargada de asegurar el leal cumplimiento de lo pactado», y a Estibans para que formase parte de la Comisión Mixta para el examen de tarifas al que se refiere el artículo 6 el Convenio. ${ }^{27}$ Por su parte, el Consejo de MZA nombró a Bauer y Maristany para suscribir el Convenio de reparto de productos y formar parte de las Comisiones mixtas a las que se refiere el artículo 6, relativo a las tarifas (para el que se destina a Maristany), y el artículo 14, relativo a las medidas para asegurar el cumplimiento del Convenio (para el que se destina a Bauer y Maristany). ${ }^{28}$

El objeto del contrato «Sindicatura general de productos» era regular el transporte combinado de ambas empresas. El acuerdo se basaba en crear un fondo común para los ingresos procedentes del tráfico en sus redes combinadas. Era un contrato público, tanto que en ocasiones el Estado tuvo que dirimir entre las partes, para reconocer deudas. El contrato pretendía buscar, para el transporte de mercancías, la ruta que resultase más económica con arreglo a las tarifas legalmente establecidas, siempre que existiera esa posibilidad.

25. El Proyecto del contrato fue firmado por MZA y NORTE el 17 de mayo de 1906.

26. Un aspecto fundamental para entender por qué se tomó este acuerdo fue un viaje a Estados Unidos realizado por Maristany. A su vuelta, escribió que en Estados Unidos, tras muchos años de competencia y de guerra de tarifas, las compañías «han preferido finalmente la coalición entre compañías a la competencia; cada día son menos frecuentes las guerras de tarifas y más permanentes las Asociaciones o los Sindicatos de tráfico» (Maristany, 1906, p. 28).

27. Acta $n .{ }^{\circ} 727$ del Libro de actas del Consejo de Administración de NORTE: 15 de junio de 1906.

28. Todo esto queda reflejado en una carta enviada por el presidente de MZA al Consejo de NORTE (Acta n. ${ }^{\circ} 728$ del Libro de actas del Consejo de Administración de NORTE: 2 de julio de 1906). 
El aspecto más importante del nuevo pacto (art. 6) consistió en la creación de una Comisión Mixta de las dos compañías para efectuar un seguimiento del Convenio. La Comisión Mixta entre NORTE y MZA se encargaría de resolver la política general de transporte combinado, de llegar a acuerdos para el transporte de mercancías específicas, de establecer las tarifas nacionales y de plantear acuerdos en sus relaciones internacionales. ${ }^{29}$

El proyecto de Convenio con MZA para la repartición de los productos del tráfico correspondientes a ambas redes fue aprobado por el Comité de Madrid de NORTE y posteriormente remitido al Comité de Barcelona de NORTE por carta el 26 de mayo de 1906. El Comité de Barcelona de NORTE escribió una carta, el 12 de junio de 1906, al Consejo en el que comunicaba la aprobación del proyecto de Convenio. ${ }^{30}$ Con ello, el proyecto de Convenio con MZA fue aprobado por los Comités de Madrid, París y Barcelona de NORTE.

El 27 de junio de 1906 se reunieron Bauer y Maristany por MZA, y Borregón y Estibans por NORTE, para firmar el Convenio, ${ }^{31}$ con el objeto de repartir entre ambas empresas los productos provenientes del tráfico de las dos redes, ateniéndose a un coeficiente basado en la proporción de los productos brutos obtenido en los años 1901-1905, ambos inclusive, de cada una de las empresas.

La primera sesión de la Comisión Mixta NORTE-MZA se celebró en el salón del Consejo de NORTE el 30 de junio de 1906. ${ }^{32}$ A partir de ese momento la Comisión Mixta gobernó conjuntamente NORTE y MZA. La Comisión Mixta supuso, en la práctica, una manifestación del grado de acuerdo a que habían llegado las dos grandes compañías, que ya funcionaban de facto como un oligopolio (es posible hablar de un duopolio en el ferrocarril español).

Los asistentes a esa primera sesión fueron Bauer, Maristany (MZA), Borregón y Estibans (NORTE), los directores generales de ambas compañías (Suss por NORTE y Waldman por MZA) y los jefes de Servicios de Intervención de NORTE, de MZA y de la red catalana. En esta reunión se examinaron los trabajos preliminares para la fijación del coeficiente a que se refiere el artículo 2 del Convenio, discutiendo minuciosamente todas las partidas que pudieran ofrecer alguna duda.

Los presentes se comprometieron a realizar una nueva reunión, el 10 de octubre de 1906, para examinar los trabajos definitivos realizados por las In-

29. Ambas compañías comenzaron a plantear acuerdos en sus relaciones internacionales, a medida que aumentaron los vínculos con los mercados exteriores, especialmente los de Europa central y Portugal. Los vínculos de la Comisión Mixta NORTE-MZA con los mercados exteriores fueron analizados por Vidal Olivares (1995a; 1995b).

30. Sesión del Comité de Barcelona: 12 de junio de 1906 y Acta n. ${ }^{\circ} 727$ del Libro de Actas del Consejo de Administración de NORTE: 15 de junio de 1906.

31. Acta n. ${ }^{\circ} 728$ del Libro de actas del Consejo de Administración de NORTE: 2 de julio de 1906.

32. AHF L 0454-0458, Actas de la Comisión Mixta entre MZA y NORTE (1906-1918). 
tervenciones para la fijación del coeficiente de reparto. ${ }^{33}$ Un signo de la importancia de los registros contables en la historia ferroviaria es que este coeficiente de reparto de productos se hace en función de los datos incluidos en el Informe Anual del año anterior.

En la reunión de 10 de octubre de 1906, Borregón comunicó al Consejo de NORTE que después de varias reuniones de la Comisión Mixta, los Servicios de Intervención de MZA y NORTE acordaron la distribución de los productos, único aspecto que faltaba por determinar. Hasta ese momento existía una diferencia, entre las partes, sobre el producto neto de la explotación, donde se manifestaba un $42 \%$ para MZA y un 58\% para NORTE. ${ }^{34} \mathrm{El}$ acuerdo final ratificado por los Comités y Consejos de ambas empresas fue de $46,588 \%$ para MZA y $53,412 \%$ para NORTE. ${ }^{35}$

El 20 de noviembre de 1906, el presidente del Consejo de MZA dirigió una carta al presidente de NORTE en la que comunicaba que en la Sesión del Consejo de MZA de 12 de octubre de 1906, se había aprobado el coeficiente de productos entre ambas compañías. ${ }^{36}$

En este proceso se observa como el Convenio es aprobado primero por NORTE, que es la que parece más interesada de las dos. Además, en la negociación tuvo que ceder una parte de su porcentaje inicial. Ambos hechos tienen sentido si se analiza la situación económica de NORTE, pues estaba en crisis. En los gráficos podemos observar que NORTE tenía menos viajeros netos (Figura 4), peores resultados contables (Figura 3), y más cargas financieras que MZA (Figura 1). Tan mala era la situación que, a principios de 1906, el director de NORTE, Gustave Bachy, había presentado un plan de ajuste de los efectivos de la plantilla y de sus sueldos. ${ }^{37}$

Las tarifas y combinaciones se adoptarían de común acuerdo. Ambas compañías acordaron que las nuevas tarifas, y las combinadas, fueran prorrateadas por kilómetros. Igual criterio se aplicaría a los billetes extraordinarios aplicados a ferias, fiestas, baños y otros eventos. Así como a la emisión de billetes de favor, de libre circulación, de caridad o de empleados y sus familias. Incluso en el caso de que surgiese otra empresa competidora, ambas sociedades considerarían sus sedes como una sola. En el caso de anexionar nuevas redes o subarrendar tráficos, ambas compañías lo comunicarían, siempre que

33. Acta n. ${ }^{\circ} 728$ del Libro de actas del Consejo de Administración de NORTE: 2 de julio de 1906.

34. Gaceta de los Caminos de Hierro, 1 de septiembre de 1906.

35. Acta n. ${ }^{\circ} 730$ del Libro de actas del Consejo de Administración de NORTE: 20 de octubre de 1906.

36. Acta n. ${ }^{\circ} 731$ del Libro de actas del Consejo de Administración de NORTE: 29 de noviembre de 1906.

37. Informe sobre cuadros de personal del director Bachy dirigido a los señores del Comité. Madrid 10 de enero de 1905. Documento incluido en AHF, E-118-4. Cuadros de personal 1905. 
desvirtuaran o modificaran las corrientes de tráfico establecidas a la firma del convenio.

La Comisión Mixta, compuesta por los consejeros delegados y los directores de las compañías, debía aprobar los proyectos de nuevas tarifas. La gestión de actuaciones comerciales complementarias de las compañías MZA y NORTE, llevó a ambas compañías a adoptar criterios de gestión de tráficos que conjugaran sus intereses. Se agruparon los repartos de los productos procedentes del tráfico, mediante la creación de un fondo común, donde se aportarían los productos netos de la aplicación de las tarifas, excluyendo cualquier otro ingreso ajeno a este concepto. Los productos que procedan de la aplicación de las tarifas para el público, formarían un fondo común; deducidos los gastos de transporte, calculados en un $30 \%$, el producto neto se distribuirá entre ambas compañías en la proporción que resulte de los productos brutos que obtuvieron en el quinquenio 1901-1905. ${ }^{38}$

La Comisión Mixta realizará un examen y aprobación de proyectos de las compañías para obligar a mejorar el material fijo y móvil de un modo uniforme para que ninguna quede perjudicada y asegurar los tráficos. Esta uniformidad se extiende a los pases y billetes de gracia. ${ }^{39}$ El hecho de que una línea

GRÁFICO 4 - Viajeros netos transportados de NORTE y MZA en millones de pesetas

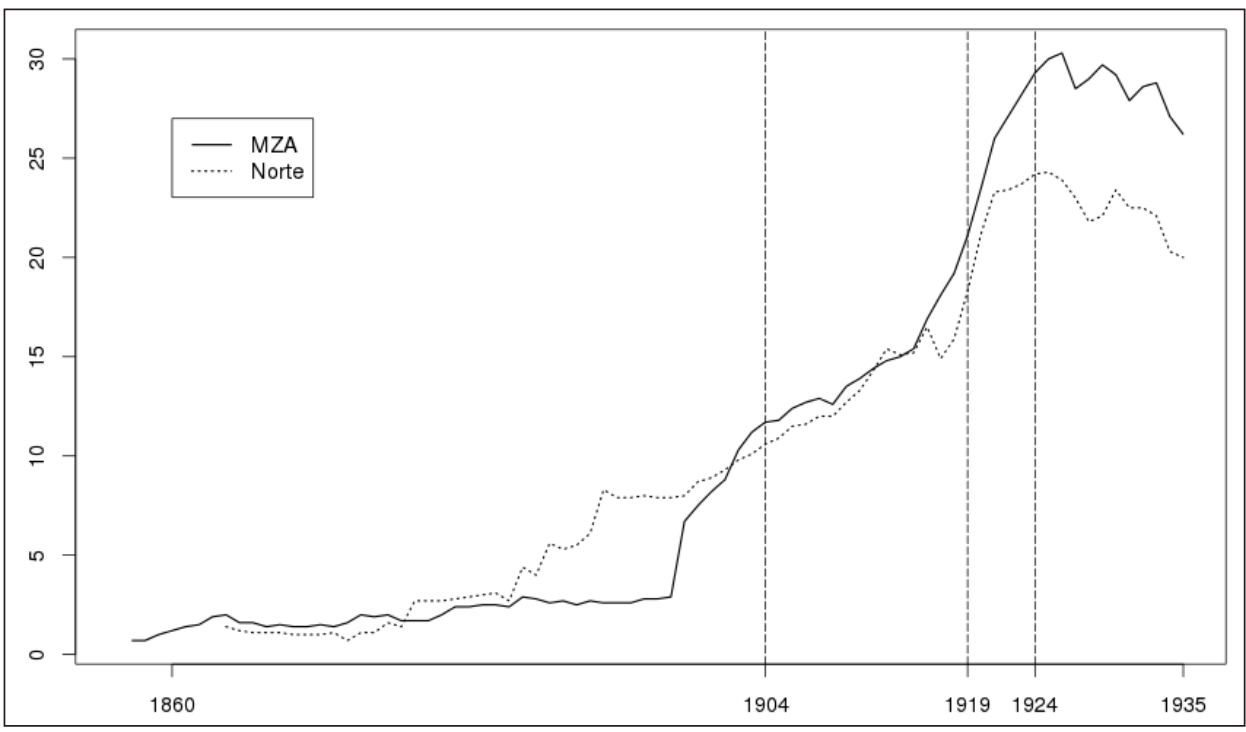

Fuente: Elaboración propia.

38. La vigilancia del Convenio se llevaría a cabo mediante la creación de un servicio de intervención recíproca, que actuaría por meses y, dentro de estos, por décadas, sobre la liquidación que cabía aplicar. Gaceta de los Caminos de Hierro, 8 de agosto de 1906.

39. $A B C$ (18 de agosto de 1906, p. 5). 
quedara interrumpida por fuerza mayor no afectaría al Convenio. Ambas compañías decidieron unificar la contabilidad para facilitar la recíproca intervención.

El acuerdo no solo benefició a las compañías, sino también al público, al aplicarse el principio obligatorio de la «distancia mínima» en los transportes, pasando a ser más rápidos y económicos. El tráfico que acudiera de una a otra red se computaría como tráfico local.

El administrador comisionado de la Red Catalana de MZA envió una carta a la Dirección de NORTE el 27 de enero de 1907 en la que se transmite el acuerdo tomado por el Consejo de Administración de MZA, respecto al Apeadero de San Andrés de la línea de Zaragoza a Barcelona de NORTE. La Comisión Mixta accede a que NORTE construya una apeadero en la línea de Zaragoza a Barcelona, junto a San Andrés del Palomar, bajo condición de que si dejara de regir el Convenio de 27 de junio de 1906, NORTE estará obligada a satisfacer a MZA el importe del producto bruto de todos los transportes que se realizasen por medio de ese apeadero sobre las vías de NORTE entre Moncada y Barcelona, una vez deducido de dicho producto el 30\% desde el día en que dejase de entrar el vigor el Convenio. El Consejo de NORTE acepta en todas las partes el acuerdo del Consejo de MZA sobre el asunto. ${ }^{40}$

En 1917, MZA solicitó la revisión del tipo del 30\% fijado en el artículo 1 del Convenio de 1906 para el abono de los gastos ocasionados por los transportes respectivamente efectuados. ${ }^{41}$ No se aprobó por la negativa del Informe del director de NORTE, del Informe de su Comité de París, de la Memoria de Robaglia, ingeniero consultor del Comité de París, y de la opinión de Coll y Portabella, representante del Comité de Barcelona. ${ }^{42}$

El presidente del Consejo de MZA enviaba cartas al Consejo de NORTE indicando que había aprobado todas las actas de la Comisión Mixta. ${ }^{43}$ Por su parte, el Consejo de NORTE aprobó todas las actas, a excepción de las adoptadas en las sesiones celebradas los días 13 y 14 de diciembre de 1906, refundiendo en uno solo los artículos 4 y 6 del contrato de 27 de junio de 1906 por

40. Acta n. ${ }^{\circ} 733$ del Libro de actas del Consejo de Administración de NORTE: 28 de enero de 1907.

41. Acta n..$^{\circ} 870$ del Libro de actas del Consejo de Administración de NORTE: 30 de noviembre de 1917, apartado III. Tras la solicitud de MZA, comenzó un proceso de delibreación dentro de la compañía NORTE y una serie de comunicaciones con MZA (Acta n. ${ }^{\circ} 872$ del Libro de actas del Consejo de Administración de NORTE: 8 de enero de 1918, apartado VII). Carta del Presidente de MZA al presidente de NORTE, fechada el 11 de marzo de 1918, y la fechada 18 de abril de 1918 (Acta n. ${ }^{\circ} 876$ del Libro de actas del Consejo de Administración de NORTE: 20 de abril de 1918, apartado IV).

42. Carta del presidente de NORTE al de MZA, fechada el 10 de abril de 1908 (Acta n. ${ }^{\circ} 873$ del Libro de actas del Consejo de Administración de NORTE: 22 de enero de 1918, apartado IV).

43. Acta n. ${ }^{\circ} 734$ del Libro de actas del Consejo de Administración de NORTE: 28 de febrero de 1907. 
estimar que debe aplazarse para más adelante esta modificación del mencionado contrato. ${ }^{44}$ Esta es la primera desavenencia de la Comisión Mixta.

La entrada en vigor de la Sindicatura posibilitó que ambas compañías realizaran multitud de irregularidades al ofrecer a los remitentes, bajo mano, precios más bajos a los establecidos en las tarifas. ${ }^{45}$

Las consecuencias de la existencia de la Comisión Mixta fueron tres: la situación económica y de gestión de NORTE mejoró, su posición común frente al Estado se fortaleció y los lazos entre las compañías fueron más fuertes para tomar posiciones comunes.

La Comisión Mixta trajo prosperidad a NORTE, porque abrió un período de bonanza que se prolongó hasta el comienzo de la Primera Guerra Mundial. Tanto es así que restituyó el dividendo a los accionistas.

En la Junta General de Accionistas de abril de 1907, los accionistas de NORTE profirieron «manifestaciones halagüeñas de los accionistas [...] en vista de la próspera situación revelada en la Memoria». ${ }^{46} \mathrm{El}$ mismo año, Barat felicita al Consejo y a la Dirección por «el acierto que han demostrado en la administración y gerencia de la compañía, acierto que la ha conducido a un estado de prosperidad por todos conceptos, y a una situación de bienestar en el personal, que es el medio más eficaz para la buena marcha del servicio y el buen crédito de la empresa». ${ }^{47}$

La situación de la caja empezó a ser bollante, lo cual hizo que quisiera mostrarla. El 28 de junio de 1907 es la primera vez que se incorpora en el Libro de actas del Consejo de Administración de NORTE la situación de la caja de NORTE, tanto en España como en París. La situación de la caja de NORTE en España el 21 de junio de 1907 arroja las siguientes cifras: 5.318.225,24 en el Banco Español de Crédito, 54.865,93 en la Caja Central, 2.797.816,4 en el Crédito Mercantil de Barcelona y 2.000.000 en el Urquijo y Compañía. La situación de la caja de NORTE en París el 19 de junio de 1907 arroja las siguientes cifras: 7.961.039,83 en el Banco Español de Crédito en francos y 1.772.332,31 en la cuenta corriente. Respecto a los títulos a disposición, hay valores de diferentes clases, por un valor nominal de 10.490 .000 pesetas en Madrid y 2.132.877,77 en París. ${ }^{48}$

Además de la prosperidad económica, el Convenio trajo más fuerza en su lucha de poder frente al Estado, sobre todo en la gestión del cobro de los cré-

44. Acta n. ${ }^{\circ} 734$ del Libro de actas del Consejo de Administración de NORTE: 28 de febrero de 1907.

45. Ortúñez (1999a: 449) supone que en esas prácticas debió de prodigarse más NORTE.

46. Acta n. ${ }^{\circ} 738$ del Libro de actas del Consejo de Administración de NORTE: 11 de mayo de 1907.

47. Acta n. ${ }^{\circ} 740$ del Libro de actas del Consejo de Administración de NORTE: 28 de junio de 1907.

48. Acta n. ${ }^{\circ} 740$ del Libro de actas del Consejo de Administración de NORTE: 28 de junio de 1907. 
ditos por transportes contra el Estado. El presidente del Consejo de Ministros consideró sus reivindicaciones como legítimas, y se comprometió a buscar los medios de atender las obligaciones y buscar los medios para evitar los retrasos en el futuro. ${ }^{49}$ Además de para defenderse del Estado, el acuerdo supuso una alianza defensiva frente a las otras ferroviariasy frente a las presiones de los sindicatos.

La Comisión Mixta fue creada para el «desarrollo de los intereses comunes de las dos compañías» (Acta n. ${ }^{\circ} 781$ del Libro de actas del Consejo de Administración de NORTE: 25 de noviembre de 1910, apartado II.4). Desde ese momento creció la colaboración con MZA, y de la cual hay muchos ejemplos.

El candidato que propone NORTE para representar a las compañías de ferrocarriles en la Comisión creada por el Ministerio de Fomento, denominada Comisión encargada de redactar el Reglamento para la aplicación de la Ley de comunicaciones marítimas (Real Orden, 18 de mayo de 1909), está consensuado con MZA (Acta n. ${ }^{\circ} 764$ del Libro de actas del Consejo de Administración de NORTE: 23 de junio de 1909: Apartado IV). En la sesión de 1 de julio de 1909, el presidente de NORTE da cuenta al Consejo de Administración del candidato elegido de acuerdo con el Consejo. El candidato elegido fue Federico de Arriaga, jefe de Servicio de lo Contencioso de MZA (Acta n. ${ }^{\circ} 765$ del Libro de actas del Consejo de Administración de NORTE: 1 de julio de 1909: apartado IV).

MZA y NORTE nombran juntos a los delegados de la Dirección General de Correos y Telégrafos (Acta de la Comisión Mixta: 4 de octubre de 1910 y Acta n. ${ }^{\circ} 781$ del Libro de actas del Consejo de Administración de NORTE: 25 de noviembre de 1910, apartado II.4).

\section{Conclusiones}

Los problemas económicos de las ferroviarias tuvieron su origen en las deficiencias estructurales, pues su negocio requería de unos altos costes iniciales de puesta en marcha, con altas inversiones en vías y material, y altas necesidades de recursos humanos. Para compensar tantos esfuerzos económicos, el negocio requería un número elevado de viajes; sin embargo, la situación económica del país era tan mala que las empresas no encontraron la demanda suficiente.

Ante la negativa situación económica general del país, la única forma que encontraron las ferroviarias para mejorar la cuenta de resultados era aumentar las tarifas o que se incrementasen las ayudas gubernamentales para la

49. Acta n. ${ }^{\circ} 724$ del Libro de actas del Consejo de Administración de NORTE: 30 de abril de 1906. 
compra de material y el mantenimiento del personal, aunque fuera a costa de incrementar la intervención estatal en ellas. Este parecía el único camino para el Estado y para ellas, y es el que se tomó.

No obstante, NORTE y MZA, como empresas privadas, intentaron otra solución para atenuar la injerencia del Estado, buscaron acuerdos que les proporcionaran la capacidad económica y financiera suficiente para sanear sus cuentas.

Entre ambas compañías existió un acercamiento estratégico y comercial que no cesó hasta su nacionalización en 1941. Esta política se materializó en cinco principales acuerdos, y algunos otros menores. Con ellos consiguieron, por un lado, evitar la competencia entre ellas permitiendo aumentar la rentabilidad al tomar una posición común frente al mercado y las presiones sindicales y, por otro, tener la capacidad de negociación suficiente frente al Estado para negociar las ayudas y tarifas.

El acuerdo más destacable es el que tomaron NORTE y MZA en 1906 pues establecieron una de las primeras joint venture entre dos empresas para dominar un sector, frente al resto de la competencia y al Estado. La relevancia del acuerdo es máxima porque, no solo suponía la unión de las dos empresas privadas más importantes de España, sino que ambas se situaban entre las doce empresas más relevantes de Europa.

\section{Base documental y bibliográfica}

\section{Expedientes de Archivo (Archivo Histórico Ferroviario)}

AHF- E-118-4 Informe sobre cuadros de personal del director Bachy dirigido a los señores del Comité. Madrid 10 de enero de 1905. Cuadros de personal 1905.

AHF-L-0454-0458, actas de la Comisión Mixta entre MZA y Norte (1906-1918).

\section{Bibliografía de la época (hasta la creación de RENFE en 1941)}

MARistany, E. (1906). «Los ferrocarriles americanos». Revista de Obras Públicas, 54, vol. 1, n. ${ }^{\circ} 1581$, pp. $15-28$.

Marquina, J. (1940). Compañía de los Caminos de Hierro del Norte de España (18591939) (vol. 1 y 2). Madrid: Espasa Calpe.

MZA (1896a). Algunos datos para juzgar acerca del Proyecto de Ley de Auxilios á las compañias de ferrocarriles. Madrid: Sucesores de Rivadeneyra.

— (1896b). Procès. Reunión del 10 de enero de 1896

- (1924). Situación del personal en la Compañía M.Z.A (1913-1933). Madrid: Sucesores de Rivadeneyra. 
NORTE (1906). Junta General de Accionistas celebrada el 20 de mayo de 1906, Memoria del Consejo de Administración del año 1905 Resoluciones de la Junta, Madrid: Imprenta Central de los Ferrocarriles.

- (1907). Junta General de Accionistas celebrada el 11 de mayo de 1907, Memoria del Consejo de Administración del año 1906, Resoluciones de la Junta. Madrid: Imprenta Central de los Ferrocarriles.

- (1924). Junta General de Accionistas celebrada el 1 de junio de 1924, Memoria del Consejo de Administración del año 1923, Resoluciones de la Junta. Madrid: Imprenta Central de los Ferrocarriles.

- (1940). Compañia de los Caminos de Hierro del Norte de España (1859-1939). Historia, Actuación, Ingresos, Gastos y Balance, vol. I. Texto y Gráficos, 2 volúmenes. Cuadros Estadísticos. Madrid: Espasa Calpe.

NORTE y MZA (1902). Contrato y reglamento celebrados entre la Compañia de los Caminos de Hierro del Norte y la de los Ferrocarriles de Madrid a Zaragoza y a Alicante para el cambio de material. Madrid: Imprenta Central de los Ferrocarriles.

- (1915). Nota sobre la influencia que en el precio de venta de los artículos de primera necesidad que se consumen en Madrid, tienen los transportes por ferrocarril. Madrid: Imprenta Alemana.

- (1916). Contestación a la exposición presentada al Excmo. Sr. Ministro de Fomento por la Comisión de Entidades Económicas de Zaragoza. Madrid.

- (1932). El problema ferroviario. Estudios realizados y medidas legislativas adoptadas en España para intentar su resolución, 1918-1932. Madrid: Gráfica Administrativa.

- (1933). Problema de los Ferrocarriles: antecedentes, datos, soluciones. Madrid: Gráfica Administrativa.

- (1934). La ordenación ferroviaria: documentos parlamentarios. (2. ${ }^{\mathrm{a}}$ ed.). Madrid: Compañías de los Ferrocarriles del Norte de España y de Madrid a Zaragoza y a Alicante.

NORTE, MZA, ANDAluCEs, MCP y OESTE (1907). Memoria referente a los créditos de las compañías de ferrocarriles contra el Estado por transportes oficiales. Madrid: Establecimiento Tipográfico Sucesores de Rivadeneyra.

ToRre, E. DE LA (1892-1935). Anuario de Ferrocarriles Españoles. Madrid: Imprenta de Alrededor del Mundo.

\section{Bibliografía moderna}

BAllesteros Doncel, E. (1997). «Una estimación del coste de la vida en España, 18611936». Revista de Historia Económica-Journal of Iberian and Latin American Economic History, año XV, n. ${ }^{\circ}$ 2, pp. 363-395.

CAmeron Rondo, E. (1961). France and the economic development of Europe, 1800-1914. Princeton: Princeton University Press.

CArreras, A. (1990). «Industrialización española: estudios de historia cuantitativa». Madrid: Espasa-Calpe. 
- (1999). «Los ferrocarriles en Europa: algunas perspectivas históricas». En: MuÑOz RUBio, M.; SAnz Fernández, J.; Vidal Olivares, J. (eds.), Siglo y medio del ferrocarril en España, 1848-1998. Madrid: Fundación de los Ferrocarriles Españoles pp. 33-54.

CARreras, A.; TAFunell, X. (1993). «La gran empresa en España (1917-1974). Una primera aproximación». Revista de Historia Industrial, n. ${ }^{\circ}$ 3, pp. 127-175.

- (1996). «La gran empresa en la España contemporánea: entre el mercado y el estado». En: Comín, F.; MARTín AceñA, P. (eds.), La empresa en la historia de España. Madrid: Civitas, pp. 73-90.

- (1997). «Spain: Big manufacturing firms between state and market, 1917-1990». En: Chandler, A.; Amatori, F.; Hikino; T. (eds.), Big business and the wealth of nations. Cambridge: Cambridge University Press, pp. 277-304.

Cobos Arteaga, F. de los; Martínez Vara, T. (2009). «Gestión del conflicto laboral en las grandes empresas. Los modelos de la dirección de las compañías ferroviarias Norte y MZA». V Congreso de Historia Ferroviaria (Palma), 14-16 de octubre.

- (2012). «Los Péreire y las Compañías de Norte de España y Midi en la definición de las conexiones ferroviarias franco-españolas 1832-1907». VI Congreso de Historia Ferroviaria. Vitoria.

Comin, F.; Martín AceñA, P.; Muñoz Rubio, M.; Vidal Olivares, J. (1998). 150 años de historia de los ferrocarriles en España. Madrid: Fundación Ferrocarriles Españoles.

Cordero, R.; MenÉndez, F. (1978). «El sistema ferroviario español». En: Artola, M. (dir.), Los ferrocarriles en España, 1844-1943. Madrid: Banco de España, vol. 1, pp. 163-341.

García Venero, M. (1948). «Historia anecdótica del ferrocarril en España». En: Cien años de ferrocarril en España, prólogo José María F. Ladreda. Madrid: Imprenta Editorial Magisterio Español, vol. 1, pp. 1-359.

Gómez MendozA, A. (1982) Ferrocarriles y cambio económico en España (1855-1913). Un enfoque de Nueva Historia Económica. Madrid: Alianza.

- (1984). Ferrocarril y mercado interior en España (1874-1913). Cereales, harinas y vinos. Madrid: Servicio de Estudios del Banco de España, vol. 1.

- (1989). Ferrocarril, industria y mercado en la modernización de España. Madrid: Espasa Calpe.

LARrinaga Rodríguez, C. (2002). Entre Irún y los Alduides. El ferrocarril del Norte y el paso de los Pirineos a mediados del siglo XIX. Irún: Casino de Irún.

López Morel, M. Á. (2005). La casa Rothschild en España. Madrid: Marcial Pons.

Martínez VARA, T. (2004). «Los costes laborales y la crisis de MZA, 1913-1935. Datos y algunas reflexiones». Revista Transportes, Servicios y telecomunicaciones, n. ${ }^{\circ} 7$, pp. 103-146.

OrtúÑEz Goicolea, P. P. (1999a). El proceso de nacionalización de los Ferrocarriles en España. Historia de las grandes compañías, 1913-1943. Tesis doctoral, Universidad de Valladolid.

- (1999b). «La configuración de la red nacional y las grandes compañías ferroviarias: Norte y MZA, 1913-1936». En: VidAl Olivares, F; Muñoz Rubio, M.; SANZ Fer- 
NÁNDEZ, J. (eds.), Siglo y medio del ferrocarril en España, 1848-1998: economía, industria y sociedad. Alicante: Diputación Provincial de Alicante, Instituto Alicantino de Cultura Juan Gil-Albert, pp. 273-297.

SANZ FERNÁNDEZ, J. (1985). «La crisis triguera finisecular: los últimos años». En: AA. VV., La España de la Restauración Política, economía, legislación y cultura. Madrid: Siglo XXI, pp. 265-310.

TedDE DE LoRCA, P. (1978). «Las compañías ferroviarias en España (1855-1935)». En: Artola, M. (dir.), Los ferrocarriles en España, 1844-1943. Madrid: Servicio de Estudios del Banco de España, vol. 2, pp. 9-354.

Vidal Olivares, J. (1995a). «Marches nationaux ou internationaux? Les compagnies de chemins de fer en Espagne et leurs connexions intemationales avec la France et le Portugal, 1850-1914». En: Merger, M.; Carreras, A.; Giuntini, A. (dirs.), Les Réseaux Européens Transnationaux, xixe-xxe siécles, quels enjeux? Nantes: Ouest Éditions, pp. 347-360.

- (1995b). «Railway companies and the diffusion of business culture in Spain». En: DAvies, M.; Wirt, D.; F. DE GoEy (eds.), Conference on Business History. Rotterdam: Center of Business History-Erasmus Universiteit, pp. 275-284.

- (1997). La formación de los directivos en la gran empresa: El caso de la Compañía de los Ferrocarriles del Norte de España, 1858-1936. Documento de Trabajo 9702. Madrid: Fundación Empresa Pública.

- (1999). «La estructura de la propiedad, de la organización y la gestión de una gran empresa ferroviaria: la Compañía de los Caminos de Hierro del Norte de España, 1858-1936». Revista de Historia Económica, año XVII, otoño-invierno 1999, n. ${ }^{\circ} 3$, pp. 623-662. Vidal Olivares, J.; Ortúñez Goicolea, P. P. (2002). «The internationalization of ownership of the Spanish railway Companies, 1858-1936». Business History, vol. 44, n. ${ }^{\circ}$, pp. $29-54$.

Wais SAn Martín, F. (1948). «Nacimiento, desarrollo y constitución de la red española». En: Cien años de ferrocarril en España (prólogo José María F. Ladreda). Madrid: Imprenta Editorial Magisterio Español, vol. 2, pp. 29-192 y pp. 241-258.

- (1987). Historia de los ferrocarriles españoles. ( $3^{\mathrm{a}}$ ed.). Madrid: Fundación de los Ferrocarriles Españoles.

- (1974). Historia de los ferrocarriles españoles (1830-1914). Madrid: Editora Nacional. 
Oligopolistic agreements between the two main companies in a sector. The case of MZA and NORTE

ABSTRACT

The Spanish railway sector has been widely studied from various perspectives, due to the important role it has played in economic progress. This research focuses on a unique aspect, in the oligopolistic agreements reached by the two most important Spanish companies in the sector. The object of this investigation is the two largest private railway companies in Spain: NORTE and MZA.

The main conclusion of the work is that, in 1906, NORTE and MZA established one of the first joint ventures in history between two companies to dominate a sector.

KEYwORDS: history, railway, NORTE, MZA

JEL CODES: L92, N73, N74

\section{Acuerdos oligopolísticos entre las dos principales empresas de un sector. El caso de MZA y NORTE}

\section{RESUMEN}

El sector ferroviario español ha sido prolíficamente estudiado desde diversos ámbitos, debido al importante papel que ha desempeñado en su contribución al progreso económico. Esta investigación se detiene en un aspecto singular como son los acuerdos oligopolísticos alcanzados por las dos empresas españolas más importantes del sector. El objeto de esta investigación son las dos mayores sociedades ferroviarias privadas de España: NORTE y MZA.

La principal conclusión del trabajo es que, en 1906, NORTE y MZA establecieron una de las primeras joint venture de la historia entre dos empresas para dominar un sector.

PALABRAS CLAVE: historia, ferrocarril, NORTE, MZA

Códigos JEL: L92, N73, N74 